\title{
Inspiratory muscle training for diaphragm dysfunction after cardiac surgery
}

\author{
Metka Kodric, MD, Roberto Trevisan, MD, Chiara Torregiani, MD, Rossella Cifaldi, MD, \\ Cinzia Longo, MD, Fabiana Cantarutti, RT, and Marco Confalonieri, MD
}

Objective: Diaphragm dysfunction is a complication of cardiac surgery with partial or absent spontaneous recovery in most cases. Surgical diaphragm plication represents the only option when symptoms persist. Because training improves functional nerve recovery after a nerve lesion, we hypothesized that early diaphragm muscle training may be beneficial.

\begin{abstract}
Methods: A prospective, randomized at 2:1 ratio, controlled trial of diaphragm training using an adjustable pressure device (Threshold; Philips Respironics Inc, Murrysville, Pa) versus no training (sham device) was performed in patients with diaphragm paralysis after major cardiac surgery. This 1-year study recruited consecutive adult patients with sniff fluoroscopy-defined diaphragm paralysis after coronary bypass, valve replacement, or both. The outcome measures were diaphragm function recovery assessed by sniff fluoroscopy, maximum inspiratory and expiratory pressures, and lung function tests.
\end{abstract}

Results: A total of 69 patients were randomized. At 12 months, 52 patients completed the study assessments, 36 in the treatment group and 16 in the control group. Inspiratory muscle training produced a significant improvement of diaphragm mobility after 12 months $(P<.001)$. Most patients in the training group $(77.78 \%)$ experienced a partial improvement $(41.67 \%)$ or achieved a complete improvement $(36.11 \%)$ versus no improvement $(87.5 \%)$ or partial recovery $(12.5 \%)$ among controls.

Conclusions: Inspiratory muscle training may improve inspiratory muscle strength and increases paralyzed diaphragm mobility. (J Thorac Cardiovasc Surg 2013;145:819-23)

Diaphragm dysfunction is a known but often underestimated complication of cardiac surgery. ${ }^{1}$ The incidence depends on the surgical procedure characteristics and the techniques used for its detection. ${ }^{2}$ Diaphragm dysfunction can prolong the need for ventilatory support and delay hospital discharge. Moreover, symptoms may extend from dyspnea on exertion to ventilatory failure, depending on the cardiopulmonary reserve of the patient and the extent of diaphragmatic involvement. ${ }^{3}$ Unilateral paralysis occurs in the majority of cases and is considered a benign and transient complication, although it may represent a serious problem for patients affected by chronic obstructive pulmonary disease (COPD). ${ }^{4}$ However, complete recovery does not always occur, and surgical intervention by diaphragm plication may represent the only option when symptoms persist after 1 year. ${ }^{5}$ Strategies to reduce postoperative pulmonary complications after noncardiac surgery, such as incentive spirometry, showed controversial results. ${ }^{6,7}$

\footnotetext{
From the Department of Pneumology, University Hospital of Cattinara, Trieste, Italy. Disclosures: Authors have nothing to disclose with regard to commercial support. Trial registry: ClinicalTrials.gov; NCT00597298 (www.clinicaltrials.gov).

Received for publication April 25, 2012; revisions received July 6, 2012; accepted for publication July 30, 2012; available ahead of print Aug 31, 2012

Address for reprints: Marco Confalonieri, MD, SC Pneumologia, Ospedale di Cattinara, Strada di Fiume 447, 34149 Trieste, Italy (E-mail: marco.confalonieri@ aots. sanita.fvg.it).

$0022-5223 / \$ 36.00$

Copyright (c) 2013 by The American Association for Thoracic Surgery

http://dx.doi.org/10.1016/j.jtcvs.2012.07.087
}

Nevertheless, it has been experimentally shown that exercise training improves functional nerve recovery after a nerve lesion. ${ }^{8}$ We hypothesize that diaphragm muscle training using an adjustable resistance device (Threshold; Philips Respironics Inc, Murrysville, Pa) may help recovery of diaphragm paralysis after cardiac surgery, avoiding the need for diaphragm plication.

\section{MATERIALS AND METHODS \\ Patients and Study Design}

A prospective, randomized at 2:1 ratio, controlled trial was performed from January 2008 to January 2009 in consecutive patients with diaphragm paralysis after major cardiac surgery. Patients were recruited from the University Hospital of Trieste. Inclusion criteria were diaphragm paralysis on chest x-ray after major cardiac surgery (eg, coronary bypass, valve replacement, or both), age 18 to 80 years, and clinical stability, including no change in medication after hospital discharge. Patients were stabilized on treatment, and drugs were titrated during in-hospital stay. Only changes to drugs, known not to affect study parameters, were permitted on a case-by-case basis. Exclusion criteria were heart failure, COPD, and known diseases causing diaphragm weakness or paralysis. A randomization plan was generated by a statistician not involved in the study using an online random permutation generator from http://www.randomization. com. The randomization assignment was provided to the recruiting physicians in sealed envelopes. The patients and the investigators devoted to the study's data analysis were blinded to the patients' treatment assignments.

\section{Assigned Interventions and Study Protocol}

An inspiratory muscle training (IMT) program with a variable pressure device (Threshold) was used. The IMT device includes a mouthpiece and 


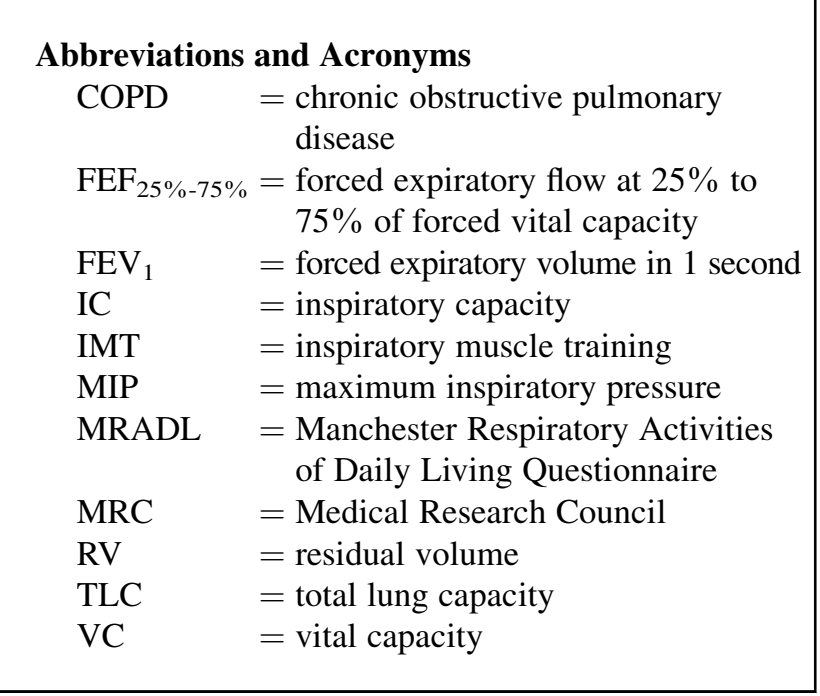

a calibrated flow-independent 1-way valve. The valve controls a constant inspiratory pressure training load, and the patient must generate the inspiratory pressure for the inspiratory valve to open and allow inhalation of air. The valve is calibrated and can be adjusted ( $2 \mathrm{cmH}_{2} \mathrm{O}$ increments) according to a percentage of the patient's maximum inspiratory pressure (MIP). A control group used nontraining physical activity by means of a sham threshold as a comparator of the true Threshold. A sham threshold was obtained by using the same device with the diaphragm removed, thus providing no resistance. Patients were evaluated by clinical examination and chest $\mathrm{x}$-ray 2 weeks after surgery; those who developed diaphragm paralysis were randomly allocated, at a 2:1 ratio, to the IMT or the control nontraining group with a sham comparator for 12 months. Interventions began within 4 weeks from surgery. Chest x-ray (in and expiratory), pulmonary function tests, assessment of dyspnea, diaphragm strength, and activities of daily living questionnaire were performed before and after interventions. MIP was measured at baseline and after 3, 6, and 12 months of treatment. The protocol was approved by the local ethics committee. All subjects signed an informed consent form. All of the evaluations were performed by investigators who were unaware of the assignment of patients to different groups. The study was registered as NCT00597298 at www.clinicaltrial.gov.

\section{Assessment of Diaphragm Motility}

Diaphragmatic motility was assessed by chest fluoroscopy with inspiratory and expiratory scans performed in the upright position. ${ }^{9}$ Patients were asked to "sniff" or quickly breathe in through their nose to detect whether both the right and left sides of their diaphragm move in the correct direction at the same time. Scans obtained after surgery were compared with baseline, and diaphragm paralysis was diagnosed with a newly observed elevation in both inspiratory and expiratory scans. The fluoroscopic "sniff" test was performed in every case. Fluoroscopy during spontaneous ventilation can demonstrate immobility or a paradoxical movement of the diaphragm, and both aspects were accepted as inclusion criteria. The interpreter of chest $\mathrm{x}$-rays was a third-party radiologist not involved in the study. At study conclusion, the same radiologic assessment was performed as for study entry. Motility recovery could be complete, partial $(50 \%$ increase in motility), or null (no change).

\section{Pulmonary Function and Respiratory Muscle Strength}

Pulmonary function values were measured by spirometry and plethysmography (SensorMedics 6200, Autobox DL; SensorMedics Corp, Yorba Linda, Calif). The analyzed parameters were vital capacity (VC), forced expiratory volume in 1 second $\left(\mathrm{FEV}_{1}\right), \mathrm{FEV}_{1} / \mathrm{VC}$ ratio, forced expiratory flow at $25 \%$ to $75 \%$ of forced $\mathrm{VC}\left(\mathrm{FEF}_{25 \%-75 \%}\right)$, inspiratory capacity (IC), total lung capacity (TLC), and residual volume (RV). Inspiratory muscle strength was assessed by measuring the MIP at RV. Expiratory muscle strength was measured as maximal expiratory pressure at TLC. The value obtained from the best of at least 3 efforts was used. All of the measurements were obtained in upright position.

\section{Inspiratory Muscle Training Protocol}

Patients were instructed to perform daily training with IMT with the Threshold inspiratory muscle device according to the following schedule: 5 ' inspiratory load set at $30 \%$ of MIP followed by 2' deep slow breathing, 5 ' inspiratory load set at $70 \%$ of MIP followed by 2 ' deep slow breathing, 5 ' inspiratory load set at $15 \%$ of MIP followed by 2' deep slow breathing, and 5 ' inspiratory load set at $80 \%$ of MIP followed by 2 ' deep slow breathing. Compliance with the training program was checked monthly by a third-party respiratory therapist not involved in the study. Inspiratory load varied between the 4 phases of each cycle, according to the percentage of MIP, and after MIP was reassessed, at months 3 and 6, the device was recalibrated. The control group followed the same schedule with a sham nontraining device.

\section{Evaluation of Dyspnea and Activities of Daily Living}

Patients were assessed for dyspnea grade at baseline and after 6 and 12 months by the Medical Research Council (MRC) dyspnea score. ${ }^{10}$ The Italian translation of the Manchester Respiratory Activities of Daily Living Questionnaire (MRADL) ${ }^{11,12}$ was used at baseline and after 6 and 12 months to evaluate the social, familiar, and occupational outcomes of the rehabilitation trial. The questionnaires were administered by the respiratory therapist in paper format, and patients ticked the score or response after reading its description. Changes from baseline of MRC and MRADL were compared between groups.

\section{End Points and Statistical Analysis}

The primary end point to determine the efficacy of treatments was diaphragm motility assessed by chest $\mathrm{x}$-ray; the secondary end point was MIP variation. Data were presented as mean \pm standard deviation. Normality was assessed by the Kolmogorov-Smirnov test. Data were analyzed by the paired $t$ test when comparing the same group at baseline and at the end of treatment period; for MIP, 1-way analysis of variance was used. Multiple comparisons adjustment was conducted by the Bonferroni test. Comparisons between groups were performed using the unpaired $t$ test. Categoric data were analyzed by the chi-square test. Statistical analysis was performed using StatView (v 5.0.1., SAS Institute Inc, Cary, NC).

\section{RESULTS \\ Patients}

Between January 2008 and January 2009, 605 patients underwent operation for coronary bypass, valve replacement, or both in the cardiac surgery unit; postoperative diaphragm dysfunction developed in 110 of these patients. In particular, 88 patients underwent coronary bypass, 9 patients underwent valve replacement, and 13 patients underwent both procedures. Twenty patients met exclusion criteria, and 21 patients refused to participate. Sixty-nine patients were randomized. Among patients randomized to IMT, 10 of them did not conclude the study: Six patients were unable to complete the training protocol, and the others had overcoming diseases (cancer, cerebral hemorrhage, and pericarditis). In the control group, 7 patients did not complete the study: Three patients were unable to complete the protocol, and the others dropped 


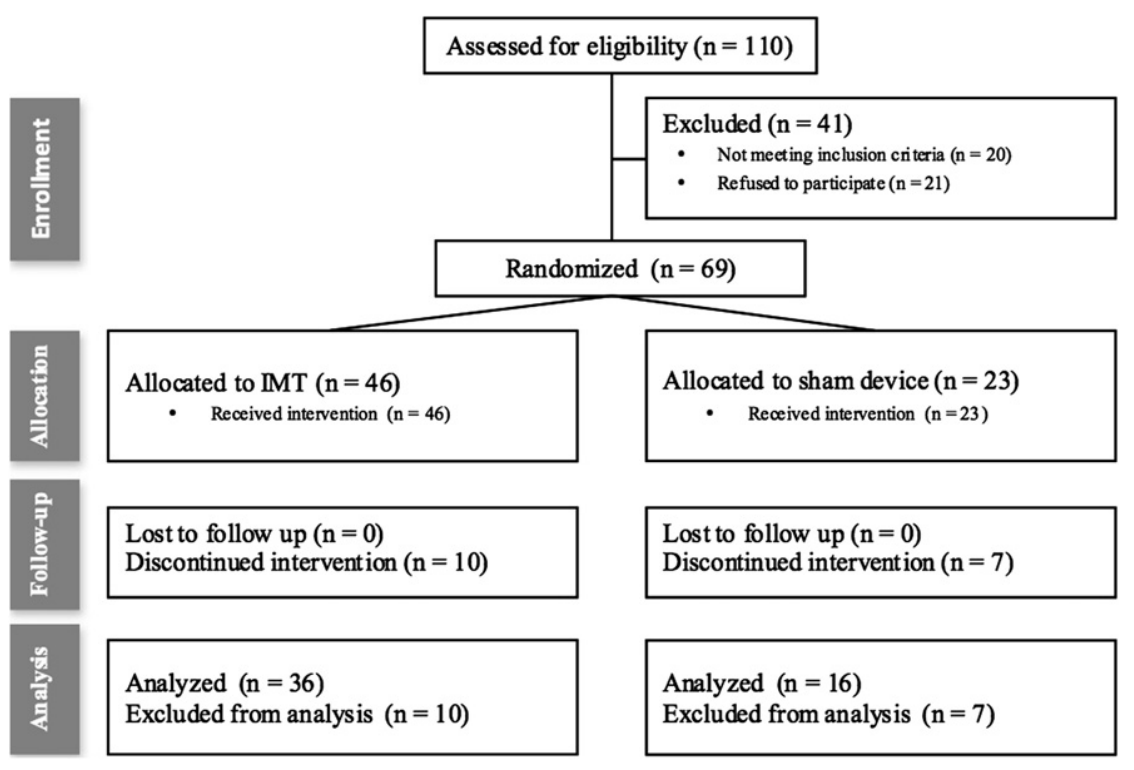

FIGURE 1. CONSORT flow chart of the study. IMT, Inspiratory muscle training.

out (adverse effects of drugs, car accident, residence variation). The 52 participants (36 IMT group, 16 controls) who concluded the trial were adherent to the protocol according to the judgment of an independent respiratory therapist. Figure 1 shows the flow diagram of the study. Patient characteristics at baseline are shown in Table 1.

Preoperative pulmonary function testing was not thoroughly available, and, when present, it was performed by different centers. However, patients did not report symptoms suggestive of diaphragm dysfunction (exertional dyspnea, dyspnea in supine position) before surgery or show diaphragm abnormalities on preoperative chest x-ray.

All procedures were performed by median sternotomy on cardiopulmonary bypass with cardioplegic arrest, with topical cold and ice slush and without the use of a phrenic protection pad. Out of 605 patients, 335 underwent dissection of the internal thoracic artery (120 patients left thoracic artery, 10 right thoracic artery, and 205 both thoracic arteries). Dissection was performed with cautery and was single in $38.8 \%$ of patients and bilateral in $61.2 \%$ of patients.

\section{Diaphragm Motility}

Three patient scans in the IMT group were not evaluated because of movement artefacts or indeterminate results. The majority of patients $(28,77.78 \%)$ in the active treatment group experienced partial improvement $(41.67 \%)$ or complete improvement $(36.11 \%)$. Most controls showed no improvement at 12 months $(87.5 \%)$; only 2 patients showed partial improvement.

\section{Respiratory Muscle Strength}

As shown in Table 2, MIP significantly improved from baseline in the active treated group $(P<.001)$, but it failed in the controls $(P=.120)$.
After 12 months, maximal expiratory pressure did not increase significantly in both groups (IMT group: $137.5 \pm$ $44.7 \mathrm{cmH}_{2} \mathrm{O}$ vs $145.1 \pm 47.3 \mathrm{cmH}_{2} \mathrm{O}, P=.065$; control group: $109.6 \pm 62.1 \mathrm{cmH}_{2} \mathrm{O}$ vs $134.3 \pm 56.4 \mathrm{cmH}_{2} \mathrm{O}$, $P=.672)$.

\section{Pulmonary Function}

VC increased in both groups from baseline to 1 year (IMT group $70.8 \% \pm 16.5 \%$ vs $86.0 \% \pm 17.1 \%$, $P<.001$; control group $67.4 \% \pm 11.2 \%$ vs $81.9 \% \pm$ $15.0 \%, P=.001) . \mathrm{FEV}_{1}$ improved from baseline in both groups, although not significantly in the control group (IMT group at baseline $66.7 \% \pm 13.7 \%$ vs $78.7 \% \pm$ $16.5 \%$ at final assessment, $P<.001$; control group at baseline $65.5 \% \pm 11.9 \%$ vs $73.6 \% \pm 13.0 \%$ at final

TABLE 1. Patient characteristics at baseline

\begin{tabular}{lccc}
\hline & $\begin{array}{c}\text { IMT group } \\
\mathbf{n}=\mathbf{3 6}\end{array}$ & $\begin{array}{c}\text { Control group } \\
\mathbf{n}=\mathbf{1 6}\end{array}$ & $\boldsymbol{P}$ value \\
\hline Gender, F/M & $6 / 30$ & $5 / 11$ & .235 \\
Age, y & $66.9 \pm 9.2$ & $68.6 \pm 9.0$ & .558 \\
$\mathrm{MIP}, \mathrm{cmH}_{2} \mathrm{O}$ & $68.0 \pm 24.9$ & $58.7 \pm 27.3$ & .233 \\
$\mathrm{MEP}, \mathrm{cmH}_{2} \mathrm{O}$ & $137.5 \pm 44.7$ & $109.6 \pm 62.1$ & .197 \\
$\mathrm{VC}, \%$ & $70.8 \pm 16.5$ & $67.4 \pm 11.2$ & .462 \\
$\mathrm{FEV}_{1}, \%$ & $66.7 \pm 13.7$ & $65.5 \pm 11.9$ & .812 \\
$\mathrm{FEV}_{1} / \mathrm{VC}, \%$ & $73.3 \pm 7.3$ & $77.5 \pm 6.2$ & .110 \\
$\mathrm{FEF}_{25 \%-75 \%} \%$ & $43.3 \pm 16.6$ & $47.6 \pm 17.6$ & .512 \\
$\mathrm{IC}_{1}, 1$ & $2.2 \pm 1.4$ & $1.6 \pm 0.7$ & .313 \\
$\mathrm{TLC}, \%$ & $68.1 \pm 12.9$ & $63.8 \pm 9.6$ & .460 \\
$\mathrm{RV}, \%$ & $68.6 \pm 22.0$ & $60.2 \pm 11.7$ & .381 \\
\hline $\mathrm{D}$
\end{tabular}

Data are presented as mean \pm standard deviation. IMT, Inspiratory muscle training $F$, female; $M$, male; $M I P$, maximal inspiratory pressure; $M E P$, maximal expiratory pres-

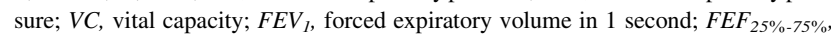
forced expiratory flow at $25 \%-75 \%$ of forced $\mathrm{VC} ; I C$, inspiratory capacity; $T L C$, total lung capacity; $R V$, residual volume. 
TABLE 2. Maximum inspiratory pressure comparison between the 2 groups during the study

\begin{tabular}{lccc}
\hline & Maximal inspiratory pressure, ${\mathbf{~} m \mathbf{H}_{2} \mathbf{O}}$ & \\
\hline & IMT group & Control group & $\boldsymbol{P}$ \\
\hline Baseline & $-68.0 \pm 24.9$ & $-58.7 \pm 27.3$ & .233 \\
$3 \mathrm{mo}$ & $-84.3 \pm 27.7$ & $-64.4 \pm 30.3$ & .025 \\
$6 \mathrm{mo}$ & $-94.4 \pm 35.5$ & $-66.8 \pm 26.1$ & .008 \\
$12 \mathrm{mo}$ & $-102.7 \pm 25.3$ & $-80.3 \pm 31.4$ & .019 \\
\hline
\end{tabular}

Control group had no IMT. Data are presented as mean \pm standard deviation. $I M T$, Inspiratory muscle training.

assessment, $P=.053)$. $\mathrm{FEV}_{1} / \mathrm{VC}$ decreased in both groups, but less in the IMT group (IMT group baseline $73.3 \% \pm$ $7.3 \%$ vs final $71.7 \% \pm 8.6 \%, P=.24$; control group at baseline $77.5 \% \pm 6.2 \%$ vs final $72.0 \% \pm 6.0 \%$, $P=.054$ ). $\mathrm{FEF}_{25 \%-75 \%}$ increased after IMT (from $43.3 \%$ $\pm 16.6 \%$ to $49.1 \% \pm 25.8 \%, P=.023)$, but decreased after sham training (from $47.6 \% \pm 17.6 \%$ to $44.0 \% \pm 21.1 \%$, $P=.425)$. TLC improved in the active treatment group from baseline to 12 months $(68.1 \% \pm 12.9 \%$ vs $76.4 \%$ $\pm 14.2 \%, P<.001)$ but did not change in controls $(63.8 \% \pm 9.6 \%$ vs $69.0 \% \pm 14.3 \%, P=.770)$. IC increased in the control group (baseline $1.6 \pm 0.71$ vs final $1.9 \pm 0.61, P=.024)$ but not in the IMT group (2.2 \pm 1.41 vs $2.2 \pm 0.71, P=.562)$. RV showed a small but insignificant increase in both groups after 12 months (IMT group $68 \% \pm 22 \%$ vs $71 \% \pm 16 \%, P=.163$; control group $60 \%$ $\pm 11 \%$ vs $61 \% \pm 19 \%, P=.329)$. At the final evaluation, there were no significant differences in pulmonary function parameters between the groups: VC $(P=.414), \mathrm{FEV}_{1}$ $(P=.356), \quad \mathrm{FEV}_{1} / \mathrm{VC}(P=.899), \quad \mathrm{FEF}_{25 \%-75 \%}$ $(P=.548)$, TLC $(P=.170)$, IC $(P=.172)$, and RV $(P=.144)$.

\section{Dyspnea Score and Activities of Daily Living}

The MRC dyspnea score significantly improved after 6 and 12 months in the IMT group compared with the control group (IMT group $-2 \pm 0.6$ from baseline at 6 months, and $-1.9 \pm 0.5$ at 12 months vs controls $-0.3 \pm 0.2$ at 6 months, and $-0.4 \pm 0.3$ at 12 months, respectively, $P=.00001$ and $P=.0001)$. The activities of daily living assessed by MRADL questionnaire improved with IMT versus controls (changes from baseline at 6 months: $+7 \pm 4$ IMT group vs $+4 \pm 2$ controls; changes from baseline at 12 months: $8 \pm$ 3 IMT group vs $5 \pm 3$ controls; respectively, $P=.007$ and $P=.002$ ).

\section{DISCUSSION}

Our study shows that IMT with the Threshold device increases diaphragm mobility and improves inspiratory muscle strength in patients with diaphragm dysfunction after cardiac surgery. Our training program also produced improvement of MRC dyspnea score in comparison with controls. To our knowledge, this is the first randomized controlled trial on the use of the Threshold device to recover diaphragm dysfunction after cardiac surgery. Mehta and colleagues ${ }^{13}$ reported a $25 \%$ to $64 \%$ recovery rate of diaphragm paralysis after a multimodality treatment, including preoperative physiotherapy. The use of IMT before coronary artery bypass grafting showed a significant decrease of postsurgical pulmonary complications in high-risk patients, ${ }^{14}$ but no study included the avoidance of diaphragm paralysis as a specifically addressed outcome of the prophylactic IMT. A retrospective uncontrolled study ${ }^{15}$ of late resistive IMT reported an improvement of greater than 400 $\mathrm{mL}$ of $\mathrm{FVC}$ in more than $50 \%$ patients with unilateral or bilateral diaphragm paralysis. The time course of recovery of the phrenic nerve may depend on the type of injury and the timing and distance over which regeneration occurs. The importance of recovering diaphragm's mobility and strength is based not only on avoiding surgery for alleviating symptoms but also on improving the patient's long-term prognosis. Respiratory muscle weakness is an independent predictor of all-cause mortality, and MIP has been associated with incident cardiovascular events, including myocardial infarction, cardiovascular death, and possibly stroke. ${ }^{16}$ These associations were independent of other measures of pulmonary function, such as FVC. In the normal condition, the diaphragm contraction contributes to $30 \%$ to $60 \%$ of the total minute ventilation, but a ventilator failure may occur in the presence of severe diaphragm dysfunction. ${ }^{15}$ With unilateral diaphragmatic paralysis, $\mathrm{VC}$ and maximum voluntary ventilation decrease from $20 \%$ to $30 \%$, with $20 \%$ reduction in oxygen uptake on the affected side. Nevertheless, it is usually well tolerated because of compensatory mechanisms, such as an increase in motor output to the intercostal muscles and the normal hemidiaphragm. ${ }^{17} \mathrm{~A}$ significant improvement in pulmonary function test results was not observed, except TLC and $\mathrm{FEF}_{25 \%-75 \%}$ with IMT. Under conditions of increased demand, a unilateral diaphragm dysfunction may cause respiratory symptoms and impairment of daily activities. ${ }^{17}$ We demonstrated that early IMT may improve symptoms and activities of daily living. We excluded those patients who might experience greater benefit to achieve clearer results. Patients with COPD were excluded because diaphragm and other respiratory muscles may undergo adaptations to chronic hyperinflation and disease, ${ }^{18}$ thus possibly altering the overall effect of IMT. In addition, patients with heart failure were excluded because their maladaptive respiratory pattern ${ }^{19}$ may have affected the perception of dyspnea. Diaphragmatic dysfunction after cardiac surgery also may occur without a clear reason. ${ }^{20}$ Surgical repair has been proposed in patients who do not show resolution of the diaphragm paralysis and have disabling respiratory compromise. ${ }^{21,22}$ Surgical plication places the paralyzed diaphragm in a position of maximum inspiration with underlying lung parenchyma 
expansion. However, there is still controversy about candidate selections and timing of surgery. On the basis of our study, we estimate that the need for surgical diaphragm plication could be decreased to no more than $5 \%$ of the total cases.

\section{Study Limitations}

We are aware that despite the great efforts to ensure study blindness, the use of sham devices is a major concern in blinded, randomized, controlled studies, both for methodological and ethical issues. ${ }^{23}$

Protocol compliance is a major issue in clinical trials and was not directly addressed in our study, although adherence to the program was checked monthly. However, Weiner and colleagues ${ }^{24}$ found relatively good compliance to the IMT program in patients with severe COPD.

The major limitation of our trial is the number of patients who were excluded from the analysis because of the inability to perform the training program. We could assume, as suggested by Guyatt and colleagues, ${ }^{25}$ that all patients lost from the treatment group did poorly and that all patients who were lost from the control group did well to verify the robustness of the results. Nevertheless, this situation is unlikely, given that there is no report in the literature showing a $100 \%$ rate of complete spontaneous recovery of diaphragm palsy after cardiac surgery, ${ }^{20}$ nor a $100 \%$ treatment failure after a rehabilitation/training program.

\section{CONCLUSIONS}

An early course of IMT with the Threshold device after cardiac surgery may promote and hasten diaphragm motility improvement, and reduce the need for surgery after 1 year. A confirmatory larger randomized controlled trial is needed.

The authors thank Mario Colomban, RT, for the enthusiastic support of this study and Dr Aniello Pappalardo for providing detailed data on cardiac surgery activity.

\section{References}

1. Dimopoulou I, Daganou M, Dafni U, et al. Phrenic nerve dysfunction after cardiac operations. Electrophysiological evaluation of risk factors. Chest. 1998;113: $8-14$.

2. O'Brien JW, Johnson SH, VanSteyn SJ, et al. Effects of internal mammary artery dissection on phrenic nerve perfusion and function. Ann Thorac Surg. 1991;52: $182-8$.
3. Stevenson JG. Effects of unilateral diaphragm paralysis on branch pulmonary artery flow. J Am Soc Echocardiogr. 2002;15:1132-9.

4. Diehl JL, Lafaso F, Deleuze P, et al. Clinically relevant diaphragmatic dysfunction after cardiac operations. J Thorac Cardiovasc Surg. 1994;107:487-98.

5. Versteegh M, Braun J, Voigt PG, et al. Diaphragm plication in adult patients with diaphragm paralysis leads to long-term improvement of pulmonary function and level of dyspnoea. Eur J Cardiothorac Surg. 2007;32:449-56.

6. Lawrence VA, Cornell JE, Smetana GW. Strategies to reduce postoperative pulmonary complications after noncardiothoracic surgery: systematic review for the American College of Physicians. Ann Intern Med. 2006;144:596-608.

7. Gosselink R, Schrever K, Cops P, et al. Incentive spirometry does not enhance recovery after thoracic surgery. Crit Care Med. 2000;28:679-83.

8. Van Meeteren NLU, Brakee JH, Hamers FTP, et al. Exercise training improves functional recovery and motor nerve conduction velocity after sciatic nerve crush lesion in the rat. Arch Phys Med Rehab. 1997;78:70-7.

9. Gierada DS, Slone MR, Fleishman MJ. Imaging evaluation of the diaphragm. Chest Surg Clin North Am. 1998;8:237-80.

10. Bestal JC, Paul EA, Garrod R, Jones PW, Wedzicha JA. Usefulness of the Medical Research Council dyspnea scale as a measure of disability in patients with chronic obstructive pulmonary disease. Thorax. 1999;54:581-6.

11. Yohannes AM, Roomi J, Winn S, Connolly MJ. The Manchester Respiratory Activities of Daily Living Questionnaire: development, reliability, and responsiveness to pulmonary rehabilitation. J Am Geriatr Soc. 2000;48:1496-500.

12. Balbi B, Marchioni M, Balestrino A, et al. Questionari per la valutazione della disabilità occupazionale in pazienti con malattie respiratorie croniche. G Ital Med Lav Erg. 2010;32:74-8.

13. Mehta Y, Vats M, Singh A, et al. Incidence and management of diaphragmatic palsy in patients after cardiac surgery. Indian J Crit Care Med. 2008;3:91-5.

14. Hulzebos EHJ, Helders PJM, Favlé NJ, et al. Preoperative intensive inspiratory muscle training to prevent postoperative pulmonary complications in high-risk patients undergoing CABG surgery. JAMA. 2006;296:1851-7.

15. Gayan-Ramirez G, Gosselink N, Troosters T, et al. Functional recovery of diaphragm paralysis: a long-term follow-up study. Respir Med. 2008;102:690-8.

16. Van der Palen J, Rea TD, Manolio TA, et al. Respiratory muscle strength and the risk of incident cardiovascular events. Thorax. 2004;59:1063-7.

17. McCool FD, Tzelepis GE. Dysfunction of the diaphragm. N Engl J Med. 2012; 366:932-42

18. Clanton TL, Levine S. Respiratory muscle fiber remodeling in chronic hyperinflation: dysfunction or adaptation? J Appl Physiol. 2009;107:324-35.

19. Hart N, Kearney MT, Pride NB, et al. Inspiratory muscle load and capacity in chronic heart failure. Thorax. 2004;59:477-82.

20. Sharma AD, Parmley CL, Sreeram G, et al. Peripheral nerve injuries during cardiac surgery: risk factors, diagnosis, prognosis and prevention. Anesth Analg. 2000;91:1358-69.

21. Celik S, Celik M, Aydemir B, et al. Long-term results of diaphragmatic plication in adults with unilateral diaphragm paralysis. J Cardiothor Surg. 2010;5:111-7.

22. Wright CD, Williams JG, Ogilvie CM, et al. Results of diaphragmatic plication for unilateral diaphragmatic paralysis. J Thorac Cardiovasc Surg. 1985;90: 195-8.

23. Fregni F, Imamura M, Chien HF, et al. Challenges and recommendations for placebo controls in randomized trials in physical and rehabilitation medicine: a report of the international placebo symposium working group. Am J Phys Med Rehabil. 2010;89:160-72.

24. Weiner P, Magadle R, Beckermann R, et al. Maintenance of inspiratory muscle training in COPD patients: one year follow-up. Eur Respir J. 2004;23:61-5.

25. Guyatt GH, Sackett DL, Cook DJ. Users' guide to the medical literature. II. How to use an article about therapy or prevention. Are the results of the study valid? JAMA. 1993;270:2598-601 